\title{
BMJ Open Instantaneous wave-free ratio guided multivessel revascularisation during percutaneous coronary intervention for acute myocardial infarction: study protocol of the randomised controlled iMODERN trial
}

Casper W H Beijnink (D , ${ }^{1}$ Troels Thim, ${ }^{2}$ Dirk Jan van der Heijden, ${ }^{3}$ Igor Klem, ${ }^{4}$ Rasha Al-Lamee, ${ }^{5}$ Jacqueline L Vos, ${ }^{1}$ Yvonne Koop (D) , ${ }^{1}$ Marcel G W Dijkgraaf (D) , ${ }^{6}$ Marcel A M Beijk, ${ }^{7}$ Raymond J Kim, ${ }^{4}$ Justin Davies, ${ }^{8}$ Luis Raposo, ${ }^{9}$ Sérgio B Baptista, ${ }^{10}$ Javier Escaned, ${ }^{11}$ Jan J Piek, ${ }^{7}$ Michael Maeng, ${ }^{2}$ Niels van Royen, ${ }^{1}$ Robin Nijveldt (D) ${ }^{1}$

To cite: Beijnink CWH, Thim T, van der Heijden DJ, et al. Instantaneous wavefree ratio guided multivessel revascularisation during percutaneous coronary intervention for acute myocardial infarction: study protocol of the randomised controlled iMODERN trial. BMJ Open 2021;11:e044035. doi:10.1136/ bmjopen-2020-044035

- Prepublication history and additional material for this paper is available online. To view these files, please visit the journal online (http://dx.doi.org/10. 1136/bmjopen-2020-044035).

Received 24 August 2020 Revised 05 December 2020 Accepted 18 December 2020

Check for updates

(c) Author(s) (or their employer(s)) 2021. Re-use permitted under CC BY-NC. No commercial re-use. See rights and permissions. Published by BMJ.

For numbered affiliations see end of article.

Correspondence to Professor Robin Nijveldt; robin@nijveldt.net

\section{ABSTRACT}

Introduction Recent randomised clinical trials showed benefit of non-culprit lesion revascularisation in STelevation myocardial infarction (STEMI) patients. However, it remains unclear whether revascularisation should be performed at the index procedure or at a later stage. Methods and analysis The instantaneous wave-free ratio (iFR) Guided Multivessel Revascularisation During Percutaneous Coronary Intervention for Acute Myocardial Infarction trial is a multicentre, randomised controlled prospective open-label trial with blinded evaluation of endpoints. After successful primary percutaneous coronary intervention $(\mathrm{PCl})$, eligible STEMI patients with residual non-culprit lesions are randomised, to instantaneous wave-free ratio guided treatment of non-culprit lesions during the index procedure versus deferred cardiac MR-guided management within 4 days to 6 weeks. The primary endpoint of the study is the combined occurrence of all-cause death, recurrent myocardial infarction and hospitalisation for heart failure at 12 months follow-up. Clinical follow-up includes questionnaires at 3 months and outpatient visits at 6 months and 12 months after primary $\mathrm{PCl}$. Furthermore, a cost-effectiveness analysis will be performed.

Ethics and dissemination Permission to conduct this trial has been granted by the Medical Ethical Committee of the Amsterdam University Medical Centres (loc. VUmc, ID NL60107.029.16). The primary results of this trial will be shared in a main article and subgroup analyses or spin-off studies will be shared in secondary papers. Trial registration number NCT03298659.

\section{BACKGROUND}

Primary percutaneous coronary intervention (pPCI) is the cornerstone of ST-elevation myocardial infarction (STEMI) treatment. ${ }^{1}$ However, approximately $50 \%$ of STEMI

\section{Strengths and limitations of this study}

- This is the first randomised controlled trial that specifically addresses non-culprit lesion treatment timing.

- Ischemia-proven non-culprit lesions receive treatment in both trial arms, potentially reducing nonnecessary treatment.

- The open-label nature of the instantaneous wavefree ratio Guided Multivessel Revascularisation During Percutaneous Coronary Intervention for Acute Myocardial Infarction facilitates treatment bias as patients in the deferred arm knowingly have non-culprit lesions which may require treatment in the future.

- The trial does not have an angiography-guided control arm, consisting of complete revascularisation without physiological guidance, although this strategy has been proven to reduce cardiovascular mortality and reinfarction.

patients have multivessel coronary artery disease (MVD/CAD), defined as non-culprit lesions with a stenosis severity $>50 \%$. $^{2}$ Patients with MVD have an increased cardiovascular morbidity, with a 2.5 -fold increase in mortality after 1 year. ${ }^{3}$ In stable CAD, it was already known that fractional flow reserve (FFR)guided revascularisation reduces the need for repeat revascularisation (HR $0.13,95 \%$ CI 0.06 to $0.30, \mathrm{p}<0.001$ ) without added procedural risk. ${ }^{4}$ However, a routine invasive strategy does not affect the hard endpoints of death, recurrent infarction or hospital admission for heart failure or cardiac arrest in stable CAD. ${ }^{5}$ 
Table 1 Studies of non-culprit lesion revascularisation versus conservative management

\begin{tabular}{|c|c|c|c|c|}
\hline Study reference & $\mathbf{n}$ & Design & $\begin{array}{l}\text { Primary outcome and } \\
\text { follow-up }\end{array}$ & Main study findings \\
\hline $\begin{array}{l}\text { Wald et al, PRAMI, } \\
\text { NEJM } 2013^{6}\end{array}$ & 465 & $\begin{array}{l}\text { Angiographic non-culprit } \\
\text { lesion } \mathrm{PCl} \text { during } \mathrm{pPCl} \\
\text { versus conservative } \\
\text { treatment. }\end{array}$ & $\begin{array}{l}\text { Cardiovascular death, } \\
\text { non-fatal MI or refractory } \\
\text { angina at } 23 \text { months. }\end{array}$ & $\begin{array}{l}\text { Non-culprit } \mathrm{PCl} \text { reduces incidence } \\
\text { of non-fatal } \mathrm{MI}(\mathrm{HR} 0.32,95 \% \mathrm{Cl} \\
0.13 \text { to } 0.75 \text { ) and refractory angina } \\
\text { ( } \mathrm{HR} 0.35,95 \% \mathrm{Cl} 0.18 \text { to } 0.69) \text { but } \\
\text { not mortality ( } \mathrm{HR} 0.34,95 \% \mathrm{Cl} 0.11 \\
\text { to } 1.08) \text {. }\end{array}$ \\
\hline $\begin{array}{l}\text { Engstrom et al, } \\
\text { DANAMI-3-PRIMULTI, } \\
\text { Lancet } 2015^{8}\end{array}$ & 627 & $\begin{array}{l}\text { FFR-guided in-hospital } \\
\mathrm{PCl} \text { of non-culprit lesions } \\
\text { versus conservative } \\
\text { treatment. }\end{array}$ & $\begin{array}{l}\text { All-cause death, } \\
\text { nonfatal Ml or repeated } \\
\text { revascularisation at } \\
12 \text { months. }\end{array}$ & $\begin{array}{l}\text { FFR-guided non-culprit PCI } \\
\text { significantly reduces } \mathrm{MACE} \text { ( } 13 \% \text { vs } \\
22 \%, \mathrm{p}=0.004) \text {, caused exclusively } \\
\text { by ischemia-driven revascularisation } \\
\text { on an individual level ( } 5 \% \text { vs } 17 \% \text {, } \\
\mathrm{p}<0.0001) .\end{array}$ \\
\hline $\begin{array}{l}\text { Smits et al, COMPARE } \\
\text { ACUTE, NEJM } 2017^{9}\end{array}$ & 885 & $\begin{array}{l}1: 2 \text { randomisation to } \\
\text { direct FFR-guided } \\
\mathrm{PCl}(\mathrm{n}=295) \text { or } \mathrm{FFR} \\
\text { measurement without } \mathrm{PCl} \\
(\mathrm{n}=590) \text {. }\end{array}$ & $\begin{array}{l}\text { All-cause death non- } \\
\text { fatal MI, repeated } \\
\text { revascularisation or } \\
\text { cerebrovascular events at } \\
12 \text { months. }\end{array}$ & $\begin{array}{l}\text { FFR-guided } \mathrm{PCl} \text { reduces the } \\
\text { combined endpoint, mainly driven } \\
\text { by less revascularisation, both } \\
\text { urgent and elective ( } 6.1 \% \text { vs } 17.5 \% \text {, } \\
\mathrm{p}<0.001) \text {. }\end{array}$ \\
\hline $\begin{array}{l}\text { Mehta et al, } \\
\text { COMPLETE, NEJM } \\
2019^{13}\end{array}$ & 4.041 & $\begin{array}{l}\text { Angiography or } \\
\text { physiology-guided } \mathrm{PCI} \\
\text { either in-hospital or } \\
<45 \text { days at the discretion } \\
\text { of the operator, versus } \\
\text { conservative treatment. }\end{array}$ & $\begin{array}{l}\text { Combined outcome of } \\
\text { cardiovascular death or } \\
\text { MI, coprimary endpoint } \\
\text { included ischemia-driven } \\
\text { revascularisation. }\end{array}$ & $\begin{array}{l}\text { Complete } \mathrm{PCl} \text { strategy resulted } \\
\text { in reduction of first coprimary } \\
\text { endpoint: } 7.8 \% \text { vs } 10.5 \%, \mathrm{p}=0.004) \text {. } \\
\text { When ischemia-driven revasc } \\
\text { was included, benefits became } \\
\text { more apparent }(8.9 \% \text { vs } 16.7 \% \text {, } \\
p<0.001) \text {. No difference was found } \\
\text { between complete treatment during } \\
\text { index admission versus a deferred } \\
\text { strategy }(p=0.62) \text {. }\end{array}$ \\
\hline
\end{tabular}

eGFR, estimated Glomerular Filtration Rate; FFR, fractional flow reserve; MACE, major adverse cardiac events; MI, myocardial infarction; $\mathrm{pPCl}$, primary percutaneous coronary intervention; PRAMI, Preventive Angioplasty in Acute Myocardial Infarction.

Contrary to stable CAD, the benefit of non-culprit lesion revascularisation differs in the setting of STEMI, as the Preventive Angioplasty in Acute Myocardial Infarction (PRAMI) trial found that angiography-guided PCI of nonculprit lesions reduced the incidence of the combined endpoint of cardiac death, nonfatal MI, and refractory angina ( $\mathrm{n}=465$, HR 0.35 , 95\% CI 0.21 to 0.58$){ }^{6}$ The CvLPRIT trial, similar in design but with a smaller sample size $(n=296)$, showed a significant reduction of urgent revascularisation in the complete PCI group. ${ }^{7}$ The addition of FFR-measurements to the study protocol yielded similar results in the more recent DANAMI-3-PRIMULTI and COMPARE ACUTE trials, reducing repeat revascularisation in the complete PCI group. ${ }^{89}$ The results of these randomised controlled trials (RCT's) are summarised in table 1 and demonstrate that non-culprit PCI, either during pPCI or later during index hospital admission, is superior to a conservative approach.

As a result, the 2017 ESC guidelines were updated to recommend that PCI of a non-culprit lesion should be considered in STEMI patients prior to hospital discharge. ${ }^{1}$ However, the most appropriate timing of non-culprit PCI remains unknown. Whether complete revascularisation should be achieved during the index procedure or as part of an outpatient approach is unclear and has not yet been evaluated in an RCT. This leads to divergent clinical practices with complete revascularisation either as index-or a staged procedure. The primary aim of the ongoing instantaneous wavefree ratio (iFR) Guided Multi-vessel Revascularisation During Percutaneous Coronary Intervention for Acute Myocardial Infarction (iMODERN) trial is to determine the optimal timing for non-culprit lesion treatment after STEMI, by comparing non-culprit PCI during the index procedure to protocolised staged PCI, guided by cardiac MR (CMR), within 4 days to 6 weeks after the index procedure. 


\section{Box 1 Inclusion criteria}

Age 18 years years and older

- Clinical presentation of ST-elevation myocardial infarction and successful primary percutaneous coronary intervention $(\mathrm{PCl})$ within 12 hours from onset of symptoms.

- One or more other, non-culprit coronary artery lesions of $>50 \%$ stenosis and feasible to be revascularised with PCI (ie, minimal diameter $2 \mathrm{~mm}$ ).

\section{Methods iMODERN trial design and population}

The trial will be described in this paper according to the 2013 Standard Protocol Items: Recommendations for Interventional Trials statement. ${ }^{10}$ Participating centres are situated throughout Europe. A total of 1146 STEMI patients will be randomised in a 1:1 ratio to iFR-guided non-culprit lesion PCI during the index procedure or to staged stress perfusion CMR-guided non-culprit lesion PCI, performed within 4 days to 6 weeks. The hypothesis of the study is that the abolishment of myocardial ischemia by also directly treating physiologically significant non-culprit coronary stenoses will lead to a reduction in the composite primary end point of all-cause death, recurrent MI, or hospitalisation for heart failure at 12 months follow-up, with a resultant reduction of healthcare costs, as compared with a delayed complete revascularisation strategy.

\section{Trial eligibility criteria}

STEMI patients with one or more non-culprit lesions amenable for PCI are included immediately following successful treatment of the culprit lesion. All eligible patients will be treated in accordance with the 2017 ESC guidelines. ${ }^{1}$ Patients with at least one $50 \%-90 \%$ (by visual estimation) non-culprit coronary artery lesion that is suitable for revascularisation with PCI, who have no exclusion criteria, will be asked for verbal consent immediately after the pPCI by the treating physician (please refer to boxes 1 and 2 for an overview of all inclusion and exclusion criteria).

\section{Box 2 Exclusion criteria}

History of ST-elevation myocardial infarction or coronary artery bypass graft.

Haemodynamic instability, respiratory failure, Killip class $\geq$ III.

- Known estimated Glomerular Filtration rate (eGFR) $<30 \mathrm{~mL} / \mathrm{min}$.

- Known contraindications for stress cardiac MR (eg, severe claustrophobia, metal implants, severe renal failure, severe asthma).

- Refusal or inability to provide informed consent.

- Life expectancy due to non-cardiovascular co-morbidity of less than 12 months months.

- Chronic total occlusion.

- Left main stem stenosis (>50\%).

- Residual non-culprit lesion in infarct coronary artery.

- Complex non-culprit target lesions.

Pregnancy.

\section{Randomisation and treatment}

Patients will be randomised using the central Syncrony EDC online random number generator, using a permuted block design per centre. Randomisation will be stratified for non-culprit lesions in the proximal or mid left anterior descending artery (LAD). A flow chart of the study treatments is provided in figure 1. If stress perfusion CMR is unfeasible for clinical or practical reasons, the physician may decide for staged iFR guided revascularisation of the non-culprit lesions.

\section{Index procedure non-culprit lesion assessment}

In patients randomised to undergo iFR-guided nonculprit lesion treatment during the index procedure, a pressure wire (Philips Volcano, Rancho Cordova, USA) assessment will be performed. Briefly, the pressure will be normalised to the aortic pressure and then the wire will be advanced past the non-culprit lesion as distal as practically possible. Then, a pressure tracing will be obtained during at least 10 cardiac cycles, after which the iFR can be calculated as the ratio between the distal pressure and the proximal pressure in the wave-free period. ${ }^{11}$ After the measurement, a drift check will be performed. If the drift value exceeds \pm 0.02 , both normalisation and iFR measurement will be repeated. By protocol, non-culprit lesions with an iFR value $\leq 0.89$ will be revascularised with PCI during the index procedure or within a bailout window of 24 hours. Figure 2 represents a case example of a patient with iFR-positive lesions, who underwent successful PCI.

\section{Staged CMR-guided non-culprit lesion assessment}

CMR will be performed between 4 days and 6 weeks after the index procedure. Clinical 1.5 or 3T scanners with a phased array cardiac receiver coil will be used. All images will be ECG-gated and acquired during mild end-expiration breath holding, using a dedicated CMR protocol. First, the long axis cine images will be acquired with a standard balanced steady-state free precession sequence. This will be followed by a test run at the basal, mid and apical short axis of the heart for the stress perfusion protocol. On correct axis planning, adenosine will be administered at $140 \mu \mathrm{g} / \mathrm{kg} / \mathrm{min}$ intravenously for $4 \mathrm{~min}$, or $210 \mu \mathrm{g} / \mathrm{kg} / \mathrm{min}$ if no haemodynamic response is observed. After $4 \mathrm{~min}$, a gadolinium-based contrast agent will be injected at $4.5 \mathrm{~mL} / \mathrm{s}$. After arrival of the contrast agent in the right ventricle, breath-hold will be performed, to acquire the saturation-recovery single-shot gradientecho sequence images. Normally, the contrast agent washes into the myocardium at a similar rate throughout the entire myocardium. However, contrast wash-in to the myocardium will be impaired in the presence of one or more coronary stenoses, observed as a hypoenhanced region amid regions that display clear contrast wash-in on a stress sequence and visually reported as a perfusion defect. Subsequently, myocardial function will be assessed completely through continuous short axis slides, covering the entire left ventricle. This will be followed by late gadolinium enhancement (LGE) imaging of all long axis and 


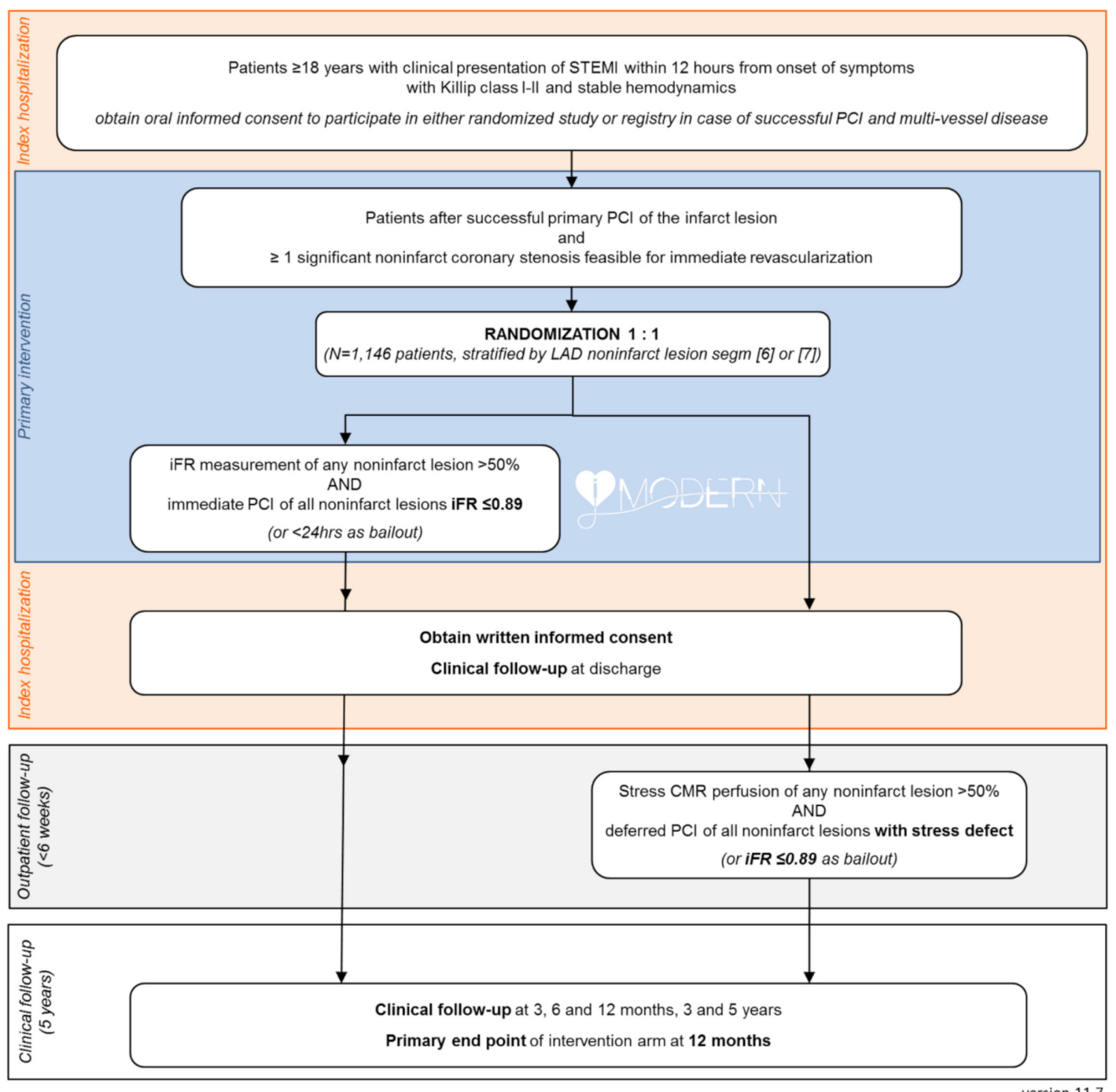

Figure 1 The study flow chart. An overview of the randomisation process, the study procedures, and patient follow-up. CMR, cardiac MR; iFR, instantaneous wave-free ratio; LAD, left anterior descending; PCI, percutaneous coronary intervention; STEMI, ST-elevation myocardial infarction.

short axis slices at $10 \mathrm{~min}$ postcontrast administration with a 2 dimensional inversion recovery, spoiled gradient echo sequence and custom optimal inversion time to correctly null normal myocardium. Finally, rest perfusion images will be acquired.

\section{Central image collection}

All angiography and CMR images will be collected centrally on a web-based platform (Webpax, Heart Imaging Technologies, Durham, USA) The iFR assessments will be made locally. A second read for quality assessment will be done by Cerebria (Cerebria, St. Albans, UK). A CMR form, containing the location of culprit and non-culprit lesions, scan details and contrast dosage, will be sent to the core lab. The core lab physician will use information from the CMR form and evaluate the coronary angiogram to assign the non-culprit lesion to a myocardial territory subtended by the poststenotic coronary artery. Then, visual interpretation of stress and rest perfusion CMR images will be performed to determine if there is ischaemia in the non-culprit lesion. Non-culprit lesions will be deemed positive if there is a perfusion defect in at least one myocardial segment, or more than $6 \%$ of the myocardium, in the territory of the designated non-culprit lesion. CMR core lab reports are used to guide revascularisation decisions and will be returned to the investigational site within 24 hours. If positive for ischaemia, patients will undergo subsequent PCI of nonculprit lesions.

\section{Follow-up}

Patients will visit the outpatient clinic at 6 months and 12 months follow-up for a clinical evaluation. This evaluation will include a brief medical history, physical examination and an ECG. Cost-effectiveness and treatment effect will be assessed through the Medical Consumption Questionnaire (iMCQ), the Productivity Cost Questionnaire (iPCQ), the Seattle Angina Pectoris Questionnaire (SAQ), EuroQoL 5-Dimension 5-Level questionnaire (EQ-5D-5L), and the Minnesota Living with Heart Failure 

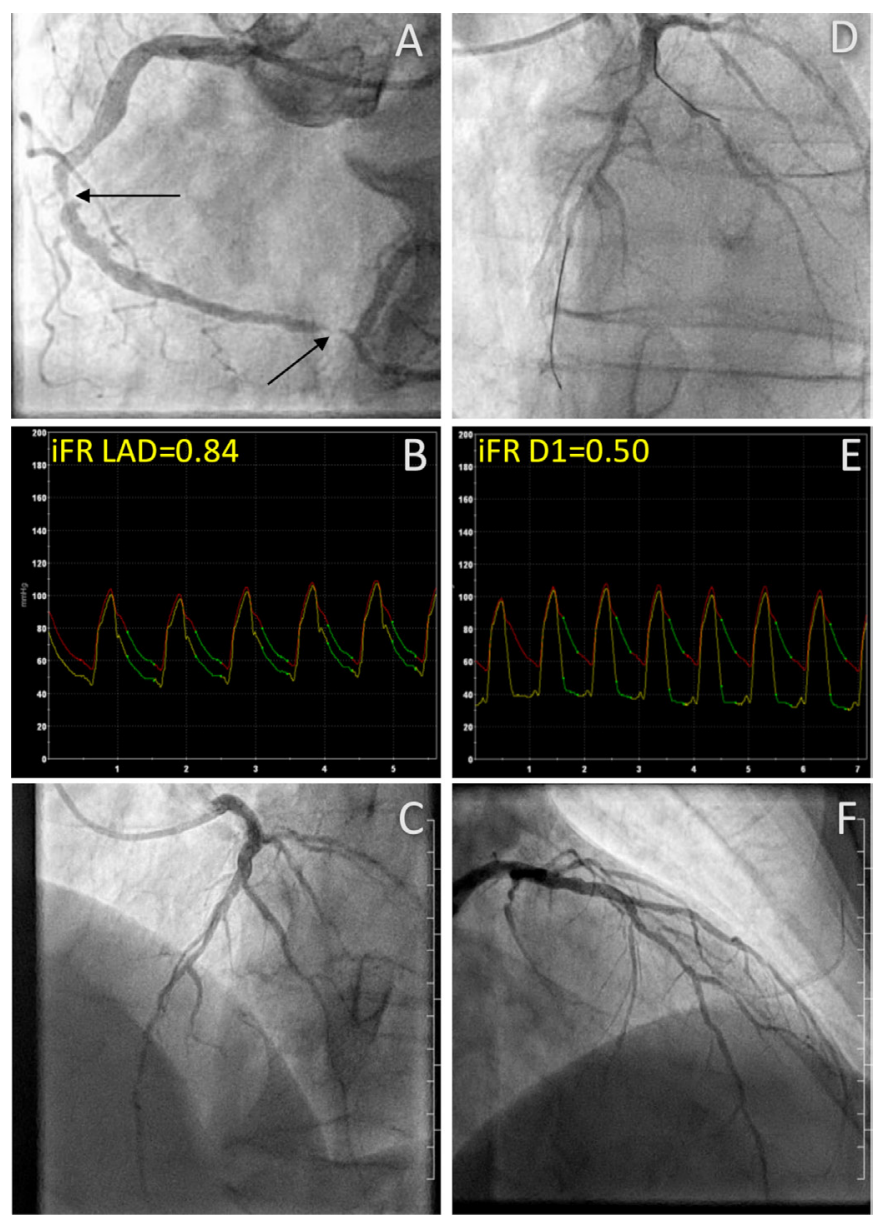

Figure 2 Direct lesion assessment. Angiographic images from a patient in the iFR-guided index procedure revascularisation arm. (A) Shows the culprit vessel, the RCA, that was treated by $\mathrm{pPCl}$ with $2 x \mathrm{DES}$ implantation at the black arrows. (D) Shows two non-culprit lesions for which the iFR value was measured ( $B$ and $E)$. Both lesions were positive for ischemia (iFR D1 $=0.50$, iFR $L A D=0.84$ ), and treated with additional $\mathrm{PCl}$ during the index procedure, after which total restoration of flow is seen on $(C, F)$. D1, first diagonal branch; DES, drug-eluting stent; iFR, instantaneous wavefree ratio; $L A D$, left anterior descending artery; $\mathrm{pPCl}$, primary percutaneous coronary intervention; RCA, right coronary artery.

Questionnaire (MLHQ). At 1 hour, 7 weeks and 3 months follow-up the EQ-5D-5L, SAQ, iMCQ and iPCQ questionnaires will be completed. At 12 months follow-up, the EQ-5D-5L, MLHQ, SAQ, iMCQ and iPCQ will be taken, to add an incremental perspective on cost and benefit. Follow-up will be completed with a telephone follow-up after 3 and 5 years. The occurrence of all-cause death, recurrent MI, all-cause hospital admission, stroke, de novo heart failure, unstable angina, stent thrombosis (defined and categorised using the criteria proposed by the Academic Research Consortium, ${ }^{12}$ repeat coronary angiography and any new unplanned revascularisation will be documented during each follow-up visit in the anonymised Syncrony EDC module. Target lesion failure will be scored, defined as treatment failure of one of the non-culprit lesions, including cardiac death, MI or clinically driven target lesion revascularisation by percutaneous or coronary artery bypass surgery. No additional blood sampling or imaging will be performed as part of the study follow-up.

\section{Patient involvement}

All enrolled patients will receive the main outcomes of this trial by mail in their native language. The results will also be shared on the trial website. Furthermore, the Dutch Heart Council will aid in the dissemination of trial results towards Dutch patient support groups.

\section{Endpoints and statistical analysis}

The combined primary endpoint is a composite of allcause death, recurrent MI, and hospitalisation for heart failure at 12 months follow-up.

Secondary endpoints include the primary end point at 6 months, cardiac death, ischaemic and haemorrhagic stroke, transient ischaemic attack, major bleeding, unstable angina, revascularisation, coronary angiography, stent thrombosis and costs related to complete, iFR-guided revascularisation versus CMR-guided treatment (cost effectiveness analysis). Target lesion failure will be documented, including cardiac death, MI or clinically driven target lesion revascularisation by percutaneous or bypass surgery at 12 months, 3years and 5 years follow-up. All outcomes will be analysed using an intention-to-treat analysis, meaning that each patient will be analysed according to the initial randomisation group, regardless of the administered treatment. Finally, a costeffectiveness and cost-utility analysis end point will be evaluated from a societal perspective with the costs per prevented cardiac events (all-cause mortality, recurrent MI and hospitalisation for heart failure) and the costs per quality-adjusted life year as the respective primary health economic outcomes. The prespecified subgroup analyses include diabetes mellitus vs no diabetes mellitus, female versus male gender, infarct lesion length terciles, infarct vessel terciles, time between onset of STEMI and PPCI, non-culprit lesion location LAD segment 6 or 7, anterior STEMI and troponin level above median versus below median.

\section{Sample size calculation}

The study is powered to assess and compare the difference in the primary end point between the iFR-guided revascularisation during index procedure group vs the CMR-guided approach after 12 months. Assuming a $16 \%$ incidence of the primary end point in the CMRguided group based on previous literature,${ }^{6} 153$ primary endpoint events are needed and should be provided by 1146 patients in order to provide the study with $80 \%$ power to detect a relative risk reduction of $35 \%$, implying an HR of 0.63 , at a two-sided alpha level of $5 \%$.

\section{Ethics and dissemination}

Permission to conduct this trial was obtained from the Medical Ethical Committee of the Amsterdam University 
Medical Centres (loc. VUmc, ID NL60107.029.16). To solve the difficulty of acquiring informed consent during an emergency procedure, the research team will provide additional information after the index procedure and during initial hospital admission. Then, written consent will be obtained in agreement with the Declaration of Helsinki.

Interim source data verification and monitoring will be performed by ongoing central monitoring of the data, remote monitoring visits and onsite monitoring visits. A medical monitor will review all adverse events to assess safety trends and risks. A clinical events committee (CEC) will be consulted in the adjudication of adverse events of interest. The CEC will assess the primary and secondary endpoints of the trial and determines whether adverse events meet protocol-specific criteria of these endpoints. A data and safety monitoring board (DSMB) will act in an advisory capacity to monitor patient safety and evaluate the progress of the trial. The DSMB will convene three times during the trial, that is, when $25 \%, 50 \%$ and $75 \%$ of patients reach the 12-month follow-up milestone. To conclude, primary results of this trial will be published in a main article. Spin-off studies and subgroup analyses will be shared in secondary papers.

\section{Current status}

Currently, there are 33 participating centres that have included 524 patients (visible in online supplemental appendix A). The names of the participating sites and their PI's are provided in online supplemental appendix A. Recruitment started in December 2017 and was expected to continue until 2021. Due to the currently ongoing COVID-19 outbreak, inclusion has been halted and follow-up visits are being done by telephone. Endpoint data will be collected as planned. Inclusion will be continued as soon as the local national public health institutes allow it and after the local medical ethics committee has been notified. As this is an international study, it is likely that inclusion will be reinitiated at different timepoints at all sites based on different local policies.

\section{DISCUSSION}

The current trial is designed to determine, with adequate statistical power to detect hard clinical endpoints, the optimal timing for non-culprit lesion PCI in patients presenting with STEMI.

The results of the recently published COMPLETE trial support that complete revascularisation in patients with STEMI and multivessel disease reduces cardiovascular death and MI. ${ }^{13}$ STEMI patients who are treated with complete revascularisation live longer and have a lower risk of a new MI. In its treatment arm, there was no difference between outcome among patients with in-hospital $(n=1353)$ or later stage PCI of non-culprit lesions $(\mathrm{n}=663)$. However, the COMPLETE trial does not answer the question whether an immediate or a delayed strategy should be chosen for non-culprit PCI, as complete revascularisation was not performed during the index procedure, a major difference with the current trial in which we speculate that immediate abolishment of non-culprit lesions improves hard outcomes. Therefore, in the aftermath of the COMPLETE trial, further specification of the right timing for non-culprit PCI in patients with STEMI constitutes an urgent research objective.

\section{Direct non-culprit lesion treatment}

There are several potential mechanisms by which the presence of stenoses in non-culprit coronary arteries may be associated with poorer outcomes in STEMI patients. At a difference with most stable patients with multivessel disease, complete revascularisation in acute STEMI with multivessel disease may have an immediate impact on cardiac function. This is because in STEMI patients there is a higher demand for flow adjacent to the infarcted myocardium to compensate for suddenly dysfunctional myocardium. Blunting of this important compensatory mechanism by physiologically significant stenoses in nonculprit vessels may lead to catastrophic consequences like pump failure, or to a more marked expression of the flowlimiting effect of the pre-existing stenoses in non-culprit vessels (ie, become ischaemia-generating under higher myocardial oxygen demand circumstances). A second mechanism leading to cardiovascular events in non-culprit territories is related to the fact that the pathobiological processes leading to plaque ulceration and thrombosis in the culprit vessel, which may be related to systemic inflammation, may be ongoing in non-culprit coronary vessels. ${ }^{14} 15$ Using optical coherence tomography, Kubo $e t$ $a l^{16}$ documented the presence of a pan-arteritis process of vulnerable coronary artery lesions after an MI. Thin fibrous caps, which are prone to plaque rupture, were present more frequently in the non-culprit lesions of acute MI patients as compared with patients with chronic CAD. Finally, non-culprit lesions may become particularly susceptible to plaque rupture by the prothrombotic milieu that occurs during an MI. ${ }^{17}$

The existence of the mechanisms described above, by which stenoses in non-culprit vessels may amplify the consequences of $\mathrm{MI}$ in the culprit-vessel territory, provide a rationale for immediate treatment of ischaemic lesions in STEMI patients, that is, during the index procedure. Indeed, a recent retrospective study suggests that immediate complete reperfusion reduces major adverse cardiac events (MACE) compared with staged reperfusion. ${ }^{18}$ Furthermore, in light of the FAME I trial, ${ }^{19}$ it is conceivable that revascularisation based on physiologic indices, that is, invasive pressure measurements or non-invasive ischemia detection, can improve outcomes as compared with revascularisation based solely on angiographic stenosis severity by reducing the need for non-necessary PCI, improving patient safety. In the current trial, iFR was chosen because it is a simpler and more cost-effective alternative to FFR with similar MACE in large prospective RCTs. ${ }^{2021}$ Moreover, the iFR is relatively stable in the 


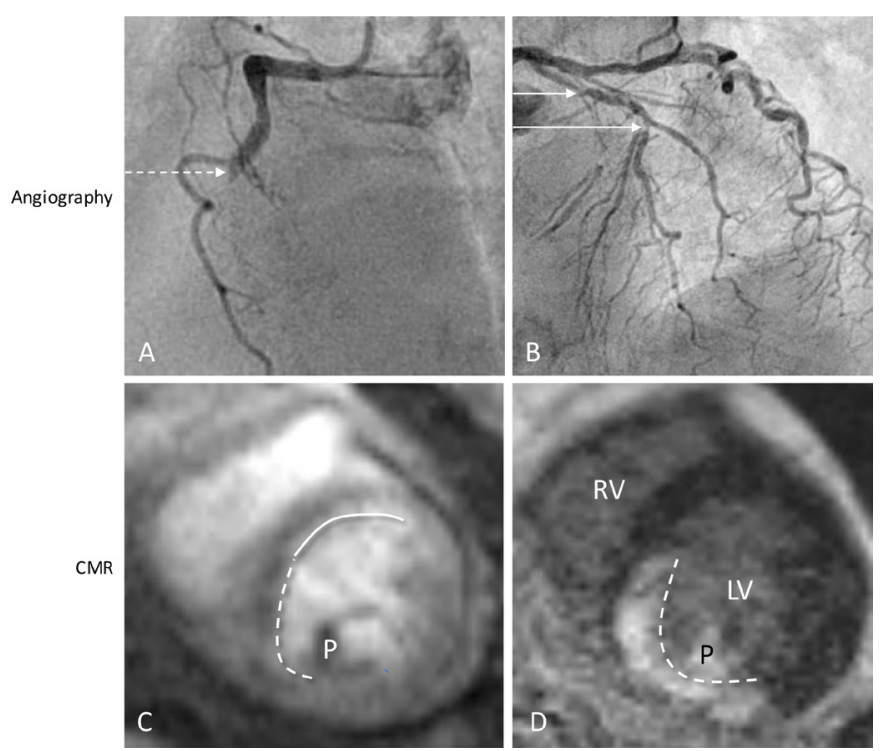

Figure 3 Typical example of a patient from the CMRguided complete revascularisation arm. (A) The dotted arrow indicates thrombus and occlusion of the RCA. (B) A nonculprit lesion of $70 \%-80 \%$ in the prox and mid LAD (solid line); (C, D) stress perfusion CMR was performed 4 weeks after primary $\mathrm{PCl}$, demonstrating two perfusion defects, of which the solid line indicates ischemia from the non-culprit LAD lesion (C), without any late gadolinium enhancement and a perfusion defect in the RCA territory caused by the subendocardial infarct ( $D$, dotted line). CMR, cardiac MR; $L V$, left ventricle; LAD, left anterior descending artery; PCl, percutaneous coronary intervention; $P$, papillary muscle; $R C A$, right coronary artery; $\mathrm{RV}$, right ventricle.

acute setting of STEMI as it is less influenced by microcirculatory disturbances, an important confounder. ${ }^{22} 23$ Additionally, iFR values as measured during index STEMI PCI procedures have a negative predictive value of $89 \%$ when compared with the iFR at a median follow-up of 16 days (IQR 5-32 days). The iFR rises significantly during this period, suggesting that it is a suitable measurement for ruling out the presence of significant non-culprit CAD. ${ }^{24}$ Finally, the use of iFR, as compared with FFR, reduces procedure times and improves experienced patient comfort, as the iFR does not require adenosine infusion. $^{20}$

\section{Cardiac CMR-guided non-culprit lesion assessment}

CMR is becoming a widely available tool that is frequently used for the assessment of patients with suspected CAD after acute MI. It is advised to perform non-invasive ischemia detection as a gatekeeper for coronary angiography, as a stepwise protocol reduces healthcare costs per quality-associated lifeyear. ${ }^{25} \mathrm{CMR}$ is a radiation-free non-invasive imaging method, allowing to combine the assessment of myocardial function, perfusion, and infarct size. In order to differentiate true ischemia from infarction, CMR offers the unique capability of setting apart a perfusion defect from irreversibly damaged myocardium by means of LGE with a high spatial resolution in one exam, as shown in figure 3. A complete CMR protocol provides this information within $30 \mathrm{~min}$. Regarding safety, a stress perfusion CMR-guided approach is non-inferior for the prevention of MACE as compared with an FFRguided approach in patients with chest pain and cardiovascular risk factors or a positive treadmill test, all without objectified CAD. This is partly because CMR-guided revascularisation significantly reduces the amount of PCI performed. $^{26}$

Technical advantages and drawbacks aside, it is known that STEMI patients with one or more perfusion defects (on SPECT) show strongly improved MACE outcomes, as well as significantly improved survival rates, when additional PCI is performed compared with optimal medical therapy alone. ${ }^{27}$ This finding is in contrast with the results of the recent ISCHAEMIA trial in which revascularisation of ischaemic lesions did not improve hard endpoints in patients with chronic $\mathrm{CAD}$, a disease that is more stable by nature. ${ }^{5}$ There is currently no randomised trial that has assessed the use of stress perfusion CMR specifically in the follow-up of STEMI. However, in the detection of ischemia in patients with suspected CAD, stress perfusion CMR outperforms SPECT in terms of sensitivity and specificity, ${ }^{28}$ especially in the setting of multivessel disease. ${ }^{29}$ Furthermore, CMR is superior to SPECT for infarct delineation, a finding that holds true especially for subendocardial MI, which SPECT detects in $28 \%$ of cases, whereas CMR does so in $92 \% .^{30}$ Finally in comparison to PET, CMR is a more widely available and less costly imaging modality with superior spatial resolution that does not subject patients to potentially harmful radiation. Given the numerous advantages of CMR over SPECT and PET, CMR is the method of choice in this trial for deferred non-culprit ischaemia detection.

\section{Trial limitations}

The iMODERN will not involve an angiography-guided treatment arm, which has been shown to be effective in reducing cardiovascular death and myocardial infarction. ${ }^{13}$ Second, the preconceived endpoint incidence as based on the results of the PRAMI trial seems too high when compared with newer, larger trials, reducing the trial's ability to detect hard outcomes. To ensure adequate power, secondary endpoint collection will also be performed at 3 and 5 years. Third, the open-label nature of the trial will mean that patients knowingly have residual $\mathrm{CAD}$ until the deferred revascularisation procedure. Any complaints of chest pain may then be linked to the residual disease, possibly resulting in earlier revascularisation than planned per study protocol.

\section{Implications}

If direct iFR-guided complete revascularisation leads to improved patient outcomes and more cost-effectiveness as compared with CMR-guided complete revascularisation, complete treatment of all obstructive coronary stenoses during the index procedure will be recommended in STEMI patients, reducing the need for additional procedures with concomitant reduction in healthcare costs. 
Author affiliations

${ }^{1}$ Cardiology, Radboudumc, Nijmegen, The Netherlands

${ }^{2}$ Department of Cardiology, Aarhus University Hospital, Aarhus N, Denmark

${ }^{3}$ Cardiology, Medisch Centrum Haaglanden, Den Haag, The Netherlands

${ }^{4}$ Cardiology, Duke University Medical Center, Durham, North Carolina, USA

${ }^{5}$ Cardiology, Imperial College London Faculty of Medicine, London, UK

${ }^{6}$ Clinical Epidemiology, Biostatistics and Bioinformatics, Amsterdam UMC - Locatie

AMC, Amsterdam, The Netherlands

${ }^{7}$ Cardiology, Amsterdam UMC Locatie AMC, Amsterdam, The Netherlands

${ }^{8}$ Faculty of Medicine, Imperial College London, London, UK

${ }^{9}$ Cardiology, Centro Hospitalar de Lisboa Ocidental EPE, Lisboa, Portugal

${ }^{10}$ Cardiology Department, Hospital Fernando Fonseca, Amadora, Portugal

${ }^{11}$ Interventional Cardiology, Hospital Clínico Universitario San Carlos, Madrid, Spain

Twitter Yvonne Koop @YvonneKoop_

Contributors The primary author CWHB was responsible for the drafting of the manuscript and the collection and interpretation of relevant sources. RN (senior author) and NvR conceived the trial protocol, acquired the necessary funding for the trial, and were coresponsible for drafting the manuscript and interpreting the used literature. The coauthors TT, DvdH, MB and RA-L are senior members of the study team, who read the article and provided substantial intellectual input. JD, LR, SB, $\mathrm{JE}, \mathrm{MM}$ and JJP are members of the steering committee, who read the article and provided important revisions to the discussion. RA-L was responsible for textual corrections. JLV, IK and RK read the article and helped to improve the Methods section. YK provided statistical advice. MGWD revised the economical analysis. All authors have read the final version of the article and agree with its content.

Funding Philips and Biotronik supported this investigator-initiated study by an unrestricted grant to the Radboudumc (no grant number). In addition, the study is financed by the Ministry of Economic Affairs of the Netherlands by a PPP allowance (grant number LSHM 16036)

Competing interests None declared.

Patient consent for publication Not required.

Provenance and peer review Not commissioned; externally peer reviewed.

Supplemental material This content has been supplied by the author(s). It has not been vetted by BMJ Publishing Group Limited (BMJ) and may not have been peer-reviewed. Any opinions or recommendations discussed are solely those of the author(s) and are not endorsed by BMJ. BMJ disclaims all liability and responsibility arising from any reliance placed on the content. Where the content includes any translated material, BMJ does not warrant the accuracy and reliability of the translations (including but not limited to local regulations, clinical guidelines, terminology, drug names and drug dosages), and is not responsible for any error and/or omissions arising from translation and adaptation or otherwise.

Open access This is an open access article distributed in accordance with the Creative Commons Attribution Non Commercial (CC BY-NC 4.0) license, which permits others to distribute, remix, adapt, build upon this work non-commercially, and license their derivative works on different terms, provided the original work is properly cited, appropriate credit is given, any changes made indicated, and the use is non-commercial. See: http://creativecommons.org/licenses/by-nc/4.0/.

\section{ORCID iDs}

Casper W H Beijnink http://orcid.org/0000-0003-1777-9586

Yvonne Koop http://orcid.org/0000-0001-6150-0859

Marcel G W Dijkgraaf http://orcid.org/0000-0003-0750-8790

Robin Nijveldt http://orcid.org/0000-0003-1530-6363

\section{REFERENCES}

1 Ibanez B, James S, Agewall S, et al. 2017 ESC guidelines for the management of acute myocardial infarction in patients presenting with ST-segment elevation: the task force for the management of acute myocardial infarction in patients presenting with ST-segment elevation of the European Society of cardiology (ESC). Eur Heart $J$ 2018;39:119-77.

2 Park D-W, Clare RM, Schulte PJ, et al. Extent, location, and clinical significance of non-infarct-related coronary artery disease among patients with ST-elevation myocardial infarction. JAMA 2014;312:2019-27

3 Sorajja P, Gersh BJ, Cox DA, et al. Impact of multivessel disease on reperfusion success and clinical outcomes in patients undergoing primary percutaneous coronary intervention for acute myocardial infarction. Eur Heart J 2007;28:1709-16.

4 De Bruyne B, Pijls NHJ, Kalesan B, et al. Fractional flow reserveguided $\mathrm{PCl}$ versus medical therapy in stable coronary disease. $N$ Engl J Med 2012;367:991-1001.

5 Maron DJ, Hochman JS, Reynolds HR, et al. Initial invasive or conservative strategy for stable coronary disease. N Engl J Med 2020;382:1395-407.

6 Wald DS, Morris JK, Wald NJ, et al. Randomized trial of preventive angioplasty in myocardial infarction. N Engl J Med 2013;369:1115-23.

7 Gershlick AH, Khan JN, Kelly DJ, et al. Randomized trial of complete versus lesion-only revascularization in patients undergoing primary percutaneous coronary intervention for STEMI and multivessel disease: the CVLPRIT trial. J Am Coll Cardiol 2015;65:963-72.

8 Engstrøm T, Kelbæk H, Helqvist S, et al. Complete revascularisation versus treatment of the culprit lesion only in patients with STsegment elevation myocardial infarction and multivessel disease (DANAMI-3-PRIMULTI): an open-label, randomised controlled trial. The Lancet 2015;386:665-71.

9 Smits PC, Abdel-Wahab M, Neumann F-J, et al. Fractional flow reserve-guided multivessel angioplasty in myocardial infarction. $N$ Engl J Med 2017:376:1234-44.

10 Chan A-W, Tetzlaff JM, Altman DG, et al. SPIRIT 2013 statement: defining standard protocol items for clinical trials. Ann Intern Med 2013;158:200-7.

11 Sen S, Escaned J, Malik IS, et al. Development and validation of a new adenosine-independent index of stenosis severity from coronary wave-intensity analysis: results of the advise (adenosine vasodilator independent stenosis evaluation) study. J Am Coll Cardiol 2012:59:1392-402

12 Garcia-Garcia HM, McFadden EP, Farb A, et al. Standardized end point definitions for coronary intervention trials: the academic research Consortium-2 consensus document. Circulation 2018;137:2635-50.

13 Mehta SR, Wood DA, Storey RF, et al. Complete revascularization with multivessel $\mathrm{PCl}$ for myocardial infarction. $N$ Engl J Med 2019;381:1411-21.

14 Joshi NV, Toor I, Shah ASV, et al. Systemic atherosclerotic inflammation following acute myocardial infarction: myocardial infarction begets myocardial infarction. J Am Heart Assoc 2015;4:e001956.

15 Han Y, Jing J, Tu S, et al. St elevation acute myocardial infarction accelerates non-culprit coronary lesion atherosclerosis. Int $\mathrm{J}$ Cardiovasc Imaging 2014;30:253-61.

16 Kubo T, Imanishi T, Kashiwagi M, et al. Multiple coronary lesion instability in patients with acute myocardial infarction as determined by optical coherence tomography. Am J Cardiol 2010;105:318-22.

17 Dutta P, Courties G, Wei Y, et al. Myocardial infarction accelerates atherosclerosis. Nature 2012;487:325-9.

18 Tovar Forero MN, Scarparo P, den Dekker W, et al. Revascularization strategies in patients presenting with ST-elevation myocardial infarction and multivessel coronary disease. Am J Cardiol 2020;125:1486-91.

19 Tonino PAL, De Bruyne B, Pijls NHJ, et al. Fractional flow reserve versus angiography for guiding percutaneous coronary intervention. N Engl J Med 2009;360:213-24.

20 Davies JE, Sen S, Dehbi H-M, et al. Use of the instantaneous Wave-free ratio or fractional flow reserve in $\mathrm{PCI}$. $N$ Engl J Med 2017;376:1824-34.

21 Götberg M, Christiansen EH, Gudmundsdottir IJ, et al. Instantaneous Wave-free ratio versus fractional flow reserve to guide PCI. $N$ Engl J Med 2017;376:1813-23.

22 van der Hoeven NW, Janssens GN, de Waard GA, et al. Tempora changes in coronary hyperemic and resting hemodynamic indices in Nonculprit vessels of patients with ST-segment elevation myocardial infarction. JAMA Cardiol 2019;4:736-44.

23 Escaned J, Ryan N, Mejía-Rentería H, et al. Safety of the deferral of coronary revascularization on the basis of instantaneous Wave-Free ratio and fractional flow reserve measurements in stable coronary artery disease and acute coronary syndromes. JACC CardiovasC Interv 2018;11:1437-49.

24 Thim T, Götberg M, Fröbert O, et al. Nonculprit Stenosis Evaluation Using Instantaneous Wave-Free Ratio in Patients With STSegment Elevation Myocardial Infarction. JACC Cardiovasc Interv 2017;10:2528-35.

25 Ge Y, Pandya A, Steel K, et al. Cost-Effectiveness analysis of stress cardiovascular magnetic resonance imaging for stable chest pain syndromes. JACC Cardiovasc Imaging 2020;13:1505-17. 
26 Nagel E, Greenwood JP, McCann GP, et al. Magnetic resonance perfusion or fractional flow reserve in coronary disease. $N$ Engl J Med 2019;380:2418-28.

27 Erne P, Schoenenberger AW, Burckhardt D, et al. Effects of percutaneous coronary interventions in silent ischemia after myocardial infarction: the SWISSI II randomized controlled trial. JAMA 2007;297:1985-91.

28 Schwitter J, Wacker CM, van Rossum AC, et al. MR-IMPACT: comparison of perfusion-cardiac magnetic resonance with singlephoton emission computed tomography for the detection of coronary artery disease in a multicentre, multivendor, randomized trial. Eur Heart J 2008;29:480-9.

29 Greenwood JP, Maredia N, Younger JF, et al. Cardiovascular magnetic resonance and single-photon emission computed tomography for diagnosis of coronary heart disease (CE-MARC): a prospective trial. Lancet 2012;379:453-60.

30 Wagner A, Mahrholdt H, Holly TA, et al. Contrast-Enhanced MRI and routine single photon emission computed tomography (SPECT) perfusion imaging for detection of subendocardial myocardial infarcts: an imaging study. Lancet 2003;361:374-9. 